\title{
Facial Expression Recognition using Patch based Gabor Features
}

\author{
Anju Chandran \\ Electronics \& Telecommunication Engg \\ Department \\ St. Francis Institute of Technology \\ Mumbai, India
}

\author{
Vaqar Ansari \\ Electronics \& Telecommunication Engg \\ Department \\ St. Francis Institute of Technology \\ Mumbai, India
}

\begin{abstract}
Facial Expression is one of the most natural, and powerful means for human beings to Communicate their emotions and intentions. The recognition of facial expressions is very important for interactive Human Computer Interfaces. One crucial step for facial expression recognition (FER) is the accurate extraction of emotional features. Numerous feature extraction techniques have been developed for recognition of expressions from static images as well as videos. This paper put forward an approach using distance features that are obtained by extracting patch based 3D Gabor features and conducting patch matching operations. The experimental results shows high correct recognition rate (CRR), fast processing time and significant performance improvements because of the consideration of facial components and muscle movements. Comparison with the state -of -the art indicates that the proposed approach achieves high CRR for JAFFE database and is one among the top performers on the CohnKanade (CK) database.
\end{abstract}

\section{General Terms}

Facial expression recognition, Human Computer Interfaces, Correct recognition Rate, Computer Vision.

\section{Keywords}

Facial components, feature extraction, Gabor filter, patch matching.

\section{INTRODUCTION}

Facial Expression Recognition (FER) is rapidly becoming an area of interest in computer vision and human computer interaction. In recent years, FER has attracted much attention because of its importance in realizing highly intelligent human-machine interfaces. Facial expressions are the most expressive way of displaying the human emotions. Human face contains significant information about emotions and the mental state of a person that can be used for nonverbal communication with computers. The fundamental objective of FER is to determine the emotional state of the face, e.g. anger, disgust, fear, happiness, sadness and, surprise regardless of the identity of the face. Even though much progress has been made in the field of FER $[1,2]$, recognition of facial expressions with high accuracy remains a difficult task due to the subtlety, complexity and variability of facial expressions [3]. FER has many potential applications in areas like human-computer interaction, behavior science, driver state surveillance and robot control [4 - 6].

Deriving an effective facial representation from original face images is a vital step for facial expression recognition. The two common approaches used to extract facial features are: geometric feature-based methods and appearance-based methods [7-10]. The geometry-based methods require accurate and reliable facial feature detection, which is very difficult to attain in real time applications. With appearancebased methods [11], image filters, such as Gabor wavelets, are applied to either the whole-face or specific face-regions to extract the appearance changes of the face. Robust recognition of facial expressions from images and videos is still a difficult task because of the difficulty in accurately extracting the useful emotional features. Facial components, especially the key components, will constantly change their positions during emotional expressions. Facial movement features are generally caused by movements of these facial components and muscles. This movement features include shape changes and feature position changes. The emotional features represent static, dynamic as well as geometric and appearance characteristics of facial expressions. That is, for a specific expression, appearance-based shape and the geometry-based position of the component feature normally change from one image to another image.

In this paper, facial movement features in static images is used to improve the performance of FER. First, patch-based Gabor features are extracted from the facial region and then performs a patch matching operation to convert the movement features into distance features. Gabor features can capture movement information very accurately, even in the case of face misalignment. In capturing regional information, they outperform the commonly used fiducial point-based Gabor and discrete Fourier transforms methods. Patch based Gabor features has shown best performance in overcoming scale, position and orientation changes. Matching scale and matching area are defined to confine the operation within a suitable space. Multi-distance values are obtained by matching patch-based Gabor features in this space. The minimum distance is selected as the final feature for emotion classification. The organization of paper is as follows: Section 2 shows the literature review. Section 3 describes the proposed methodology with detailed description of building distance features and feature selection. Section 4 shows the experimental results and comparison with the state-of-the-art performance. Section 5 ends with a conclusion.

\section{LITERATURE REVIEW}

Over the past decades many methods have been proposed for human facial expression recognition from static images to image sequence. Paul Ekman and Friesen through their researches $[12,13]$ identified six basic emotions that can be identified from facial expressions with high accuracy. These six basic facial expressions (emotions) are Happy, Surprise, Disgust, Fear, Sad and Angry as shown in figure 1. 

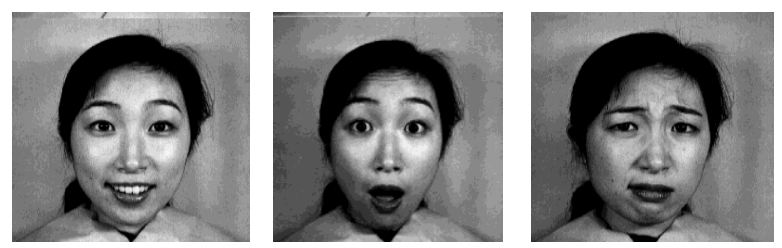

Happy

Surprise

Disgust
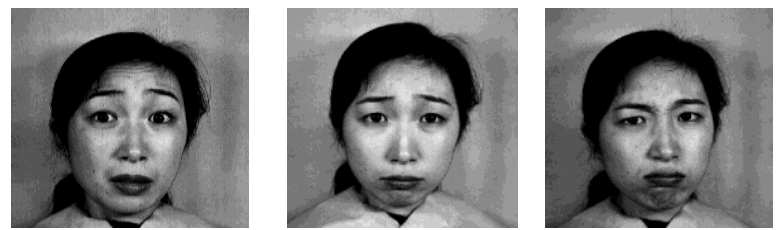

Fear

\section{Anger}

Fig.1. Six Fundamental facial Expressions

Facial motions are fundamental elements for the facial expressions recognition. The Facial Action Coding System (FACS), developed by Ekman and Friesen [14], is the most versatile and widely used method in facial expression recognition. FACS enables facial expression analysis by identifying the facial muscles which cause changes in the facial expression. This system consists of 46 Action Units to describe the facial behaviours. Even though they achieved good results, these methods often require accurate location and tracking of facial components, which is a tedious task. Principal Component Analysis (PCA), [15] also known as the Eigen face approach is one of the most commonly used methods for facial expression recognition. This technique reduces the dimensionality of image and contributes effective face indexing and retrieval. Mase and Pentland [16] used dense optical flow to estimate the activity of the facial muscles. Essa et al. [17, 18] developed an automated system to describe the facial motions, using optical flow integrated with a physical model of face muscles. Fisher's Linear Discriminant Analysis is another approach [19] that reduces the projected sample scattering and has better performance than PCA. Independent Component Analysis (ICA) [20] produces statistically independent basis vector and gives better performance than PCA, but it is computationally more expensive.

Tian et al. [21] proposed a system based on detailed parametric models of the facial features to recognize upper and lower facial action units (AUs). Jyh-Yeong et al. presented automated facial expression recognition system [22] using neural network classifiers. They used Action Units (AU) to describe the basic movements of face muscles and Rough Contour Estimation Routine (RCER) to get features of eyebrows, eyes and mouth. Hong-Bo et al. developed a facial expression recognition system [23] based on Gabor features using a local Gabor filter bank. This method uses Gabor coefficients of the fiducial points for the classification of human emotions. Caifeng Shan et al. proposed a system [24] that works against changes in illumination and takes less computation time compared to other existing systems. They used Linear Binary Pattern for feature extraction and Support Vector Machine (SVM) for classification. Luiz S. OLiveira et al. developed a system [25] using 2 Dimension Principal Component Analysis for feature extraction and K-Nearest Neighbor for classification. 2DPCA works on the whole image while PCA works on vector image. But with 2DPCA

coefficient are more so, feature selection is needed for classification.

Majority of the work done in the field of facial expression recognition do not consider the dynamics of facial movement features. These efforts consider either the geometric features of tracked facial points like, facial animation parameters, shape vectors or appearance difference between holistic facial regions in consequent frames such as optical flow, and differential-AAM. Other appearance based features such as local binary patterns (LBP), Haar, Histogram Oriented Gradients (HOG), have shown good performance in the field of FER. But they lack the capability of capturing facial movement features with high accuracy. Because these appearance based features are based on static values extracted from facial sub regions. Some methods use texture and motion changes in local facial regions such as motion units, and animation units. The above mentioned approaches on FER have not fully considered the features of facial component and muscle movements into account.

\section{METHODOLOGY}

Facial expression recognition system presented here is composed of pre-processing, training, and testing stages. During pre-processing, facial regions are cropped from database images and scaled to a resolution of $48 * 48$ pixels. Then, multiresolution Gabor images are obtained by convolving the scaled facial regions with eight scale, fourorientation Gabor filters. At the training stage, by moving a series of patches with different sizes across the training Gabor image a whole set of patches is extracted. Then the extracted patches are converted into distance features by a patch matching operation. The matching area and matching scale are defined to increase the matching space. The minimum rule is used to select the best matching feature in this space. Among the converted distance features, a set of "salient" patches is selected by Greedy feature selection method. At the test stage, using the "salient" patches the same patch matching operation is performed on a new image. The resulting distance features are fed into a multiclass support vector machine (SVM) to recognize the six basic emotions, like anger (AN), disgust (DI), fear (FE), happiness (HA), sadness (SA), and surprise (SU).

In this work, 2D Gabor filter [26] is used and it can be mathematically expressed as

$$
\begin{aligned}
& F(x, y)=\exp \left(-\frac{X^{2}+\gamma^{2} Y^{2}}{2 \sigma^{2}}\right) \times \cos \left(\frac{2 \pi}{\lambda} X\right) \\
& X=x \cos \theta+y \sin \theta, Y=-x \sin \theta+y \cos \theta,
\end{aligned}
$$

Where $\theta$ is the orientation, $\sigma$ the effective width, $\lambda$ the wavelength, and $\gamma=0.3$ the aspect ratio. Eight scales $(5: 2: 19)$ and four orientations (-45, 90,45,0 degrees) are used here. This paper directly uses the LIBSVM [27] implementation of SVMs. The one-against-the-rest strategy is used to solve the six emotion class problem. 


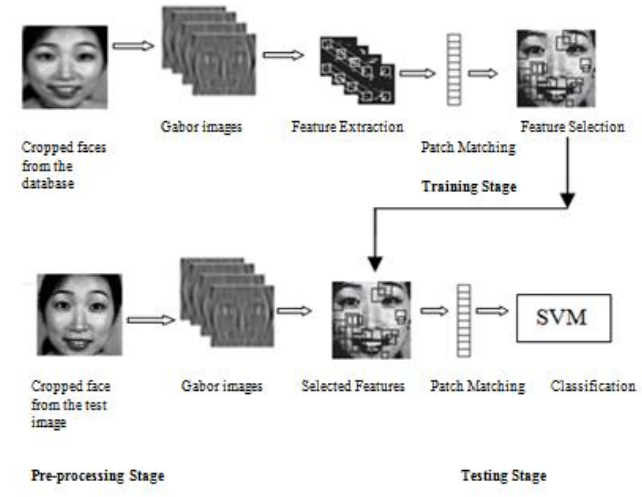

Fig.2. The proposed Framework

Building the distance features comprises of two processes: a) feature extraction and b) patch matching operation. During Feature extraction a set of discriminating 3D patches are collected for all emotions, whereas in patch matching operation these patches are converted to distance features that can capture facial movement features.

\subsection{Feature Extraction}

First of all, the training images are classified into 10 sets each of which includes images of one subject (person).Here four different patch sizes are used $2 * 2,4 * 4,6 * 6,8 * 8)$. Gabor images of four orientations and eight scales i.e. (32 Gabor images) and six emotions of each subject are considered. For each emotion $E_{k}$, where $(k=1, \ldots, 6)$ each Gabor scale $G_{s}$, where $(\mathrm{s}=1, \ldots, 8)$ and for each patch size $P_{j}$ where $(j=1, \ldots, 4)$, one Gabor image is randomly selected from all the Gabor images. The pseudo code of building distance features is shown in fig.3.

Image set $\mathrm{Si}(\mathrm{i}=1, \ldots, 10)$ with Image width $\mathrm{W}=48$ and height $\mathrm{H}=48$. Patch size $\mathrm{P}_{\mathrm{j}}(\mathrm{j}=1, \ldots ., 4)$

Emotion index $E_{k},(k=1, \ldots, 6)$, Gabor scale $G_{s}(s=1, \ldots ., 8)$

Orientation $\mathrm{O}_{\text {num }}($ num $=1, . ., 4)$

For each emotion and for each Gabor scale Choose one set $\mathrm{S}_{\mathrm{i}}$ randomly from 10 sets Moving step $=\mathrm{P}_{\mathrm{j}} / 2$.

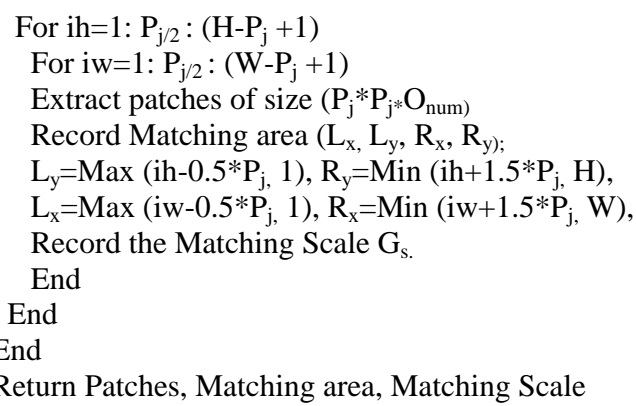

Fig.3. Pseudo code of building distance features

A patch $\mathrm{P}_{\mathrm{a}}$ is given with a size of $\left(\mathrm{P}_{\mathrm{j}} * \mathrm{P}_{\mathrm{j}} * \mathrm{O}_{\text {num }}\right)$, where $\mathrm{O}_{\text {num }}$ is the orientation number $(n=1, \ldots, 4)$. A set of $3 \mathrm{D}$ patches can be extracted by moving this patch across the row and column pixels of this Gabor image as shown in fig 4. Third, record the matching area and matching scale (Fig. 3). Finally, constitute a patch set is by combining the extracted patches of all the six emotions, all eight scales, and all four patch sizes. To increase the processing speed and to reduce the feature dimension, only a part of the patches are extracted by moving the patch $\mathrm{P}$ with a step. The moving steps are set to $1,2,3$, and 4 to four patch sizes of $2 * 2,4 * 4,6 * 6$, and $8 * 8$ respectively. With facial images of size $48 * 48$, four-orientation and eight-scale Gabor filters, the final set contains 148,032 patches. This method reserves both the location and multiresolution information of patches for expression recognition, resulting in an equal set of patches for each expression.

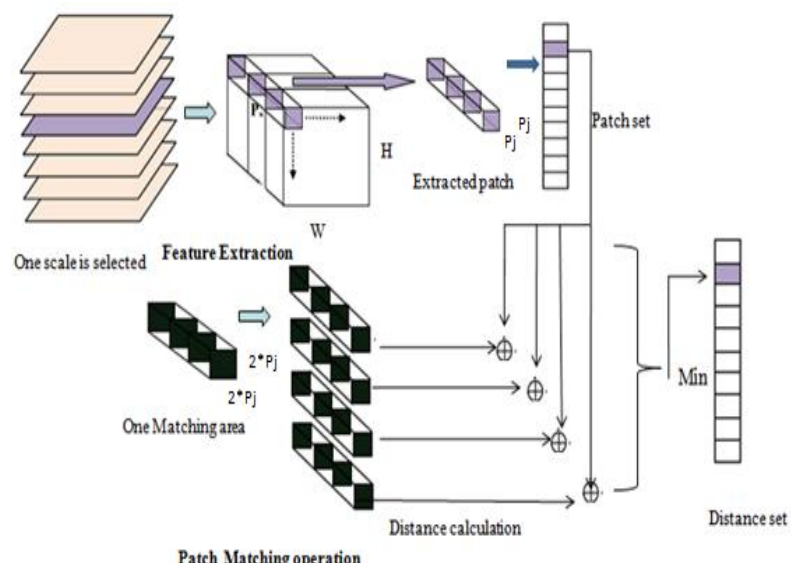

Fig.4. Distance feature formation

\subsection{Patch Matching Operation}

The patch matching operation consists of four steps. First, in order to get a bigger matching space, the matching area and matching scale are defined. This helps to accurately capture the scale and position changes due to the movements of facial features. Specifying a larger scale and a bigger area for each patch ensures the invariance to scale and position changes during the patch matching operation. The matching area of a patch $P_{a}$ with a size of $P_{j} * P_{j} * O_{n u m}$, is set to double the size of $\mathrm{P}_{\mathrm{a}}$ in width and height, but with the same center point and orientation number $\mathrm{O}_{\text {num. }}$. That is, Area $=\left(2 * \mathrm{P}_{\mathrm{j}}\right) *\left(2 * \mathrm{P}_{\mathrm{j}}\right) * \mathrm{O}_{\text {num }}$. The cropped facial regions generally belong to the same scale, so the matching scale remains the same with that of $\mathrm{P}_{\mathrm{a}}$. Second, distances are calculated by matching this patch with all patches within its matching space in the training images. Two patches are taken as inputs and one distance value is calculated based on a distance metric. The distance metric is used to evaluate the similarity between two patches and can be mathematically expressed as

$$
D L_{2}:\left\|P_{b}-P_{c}\right\|=\frac{1}{P_{j} * P_{j} * O_{n u m}} \sqrt{\sum_{i=1}^{p_{j}} \sum_{j=1}^{p_{j}} \sum_{o=1}^{O_{n u m}}\left(P_{b}^{i j o}-P_{c}^{i j o}\right)^{2}}
$$

Where, $\mathrm{P}_{\mathrm{b}}$ and $\mathrm{P}_{\mathrm{c}}$ represent two patches, $P^{i j o}$ represents the pixel values in the $i$ th row, $j$ th column, and $o$ th orientation of the patches. The distances are normalized by dividing the distance value with the number of pixels in the patches. The dense distance takes into account all orientations of each pixel in one patch. Once the distances are obtained, third step is to choose the minimum distance as the distance feature of that particular patch. Finally, combine the distance features of all the patches into a final set of 148,032 elements.

\subsection{Feature Selection}

The feature extraction generates a feature set containing 148,032 patches. Many of these features are insignificant or redundant. Unnecessary features make generalization more 
difficult and increase the size of the search space. In addition, the risk of over fitting is higher with more no of features. So it is necessary to select a subset of relevant patches in order to reduce the redundant information and feature dimension. Feature subset selection aims to select the feature that maximizes the classification accuracy. There should be a trade-off between the quality of generated feature subset solutions and the computational time. In this work the widely used Greedy feature selection method [28] which is a common example of wrapper methods is used for salient patch selection.

\subsection{Classification}

In this paper, SVM which is the most widely used machine learning algorithm is used for classification. SVMs are supervised learning models used for classification and regression. SVM algorithm is based on the statistical learning theory. It can classify the unknown data correctly due to its higher generalization ability. SVM [29] uses a linear hyper plane to separate the data set optimally in a higher dimensional plane when there is a non-linear relation between data and the input plane. If the data is linearly inseparable, a kernel transformation is used to project the data into a new space and then the optimization is carried out in the kernel space. Kernel transformation gives the inner product between two points in a suitable high dimensional space with a reduced computational cost.

\section{EXPERIMENTAL RESULTS}

\subsection{Database}

The JAFFE database [30] consists of 213 gray images of seven facial expressions posed by 10 Japanese females. These7 expressions include six basic expressions and one neutral expression .Each object has three or four frontal face images with image resolution $256 * 256$ pixels. Each image has been rated on 6 emotion adjectives by 60 Japanese subjects. In this work, 180 images of six basic expressions in the JAFFE database are used. For CK database [31], 720 images that represent one of the six basic expressions are selected, three peak images for each expression. Image resolution is $640 * 480$ or 490 pixels. For JAFFE database, the images of 10 subjects are classified into 10 sets with images of one subject per set. In the same way, all the images in the CK database are classified into 10 similar sets with all images of one subject in the same set.

\subsection{Result}

The performances results are calculated by averaging the correct recognition rate (CRR) of all sets using 10 leave-oneset out cross validations. The entire database is divided into ten equal sets. At the beginning first nine sets of the data are used for training and the remaining one set is used for testing. The procedure is repeated nine more times for different sets of training and testing data. Overall CRR of JAFFE database is $87.22 \%$ and of CK database is $96.80 \%$.

Table 1. Confusion Matrix on the JAFFE database

\begin{tabular}{|l|c|c|c|c|c|c|l|}
\hline & AN & DI & FE & HA & SA & SU & $\begin{array}{l}\text { Over } \\
\text { all }\end{array}$ \\
\hline AN & $\mathbf{2 7}$ & 1 & 0 & 0 & 1 & 1 & $\mathbf{9 0 . 0 0 \%}$ \\
\hline DI & 0 & $\mathbf{2 6}$ & 1 & 0 & 3 & 0 & $\mathbf{8 6 . 6 7 \%}$ \\
\hline FE & 0 & 3 & $\mathbf{2 3}$ & 1 & 2 & 1 & $\mathbf{7 6 . 6 7 \%}$ \\
\hline
\end{tabular}

\begin{tabular}{|l|c|c|c|c|c|c|c|}
\hline HA & 0 & 1 & 0 & $\mathbf{2 8}$ & 1 & 0 & $\mathbf{9 3 . 3 3 \%}$ \\
\hline SA & 0 & 1 & 1 & 1 & $\mathbf{2 7}$ & 0 & $\mathbf{9 0 . 0 0 \%}$ \\
\hline SU & 0 & 1 & 2 & 1 & 0 & $\mathbf{2 6}$ & $\mathbf{8 6 . 6 7 \%}$ \\
\hline
\end{tabular}

Table1. Shows the confusion matrix of six emotions on the JAFFE database. From the table it is clear that fear is the expression that is most difficult to recognize correctly. Regarding the misrecognition rate, disgust and sadness contributes the most.

Table 2. Confusion Matrix on the CK database

\begin{tabular}{|c|c|c|c|c|c|c|c|}
\hline & AN & DI & FE & HA & SA & SU & $\begin{array}{l}\text { Over } \\
\text { all }\end{array}$ \\
\hline AN & 117 & 1 & 2 & 0 & 0 & 0 & $\mathbf{9 7 . 5 2 \%}$ \\
\hline DI & 1 & $\mathbf{1 1 2}$ & 2 & 1 & 2 & 2 & $\mathbf{9 3 . 3 3 \%}$ \\
\hline FE & 0 & 4 & $\mathbf{1 1 5}$ & 0 & 1 & 0 & $\mathbf{9 5 . 8 3 \%}$ \\
\hline HA & 0 & 1 & 1 & $\mathbf{1 1 8}$ & 0 & 0 & $\mathbf{9 8 . 3 3 \%}$ \\
\hline SA & 1 & 1 & 2 & 0 & $\mathbf{1 1 6}$ & 0 & $\mathbf{9 6 . 6 6 \%}$ \\
\hline SU & 0 & 0 & 1 & 0 & 0 & $\mathbf{1 1 9}$ & $\mathbf{9 9 . 1 6 \%}$ \\
\hline
\end{tabular}

Table 2. Shows the confusion matrix of six emotions using the CK database. Surprise performs the best with a CRR of 99.16 percent. Disgust is the most difficult facial expression to be correctly recognized. Regarding the misrecognition rate, fear contributes the most

Table 3. demonstrates the comparison of the results of the proposed method with the results of the other benchmarked approaches. As shown in the table 3. The proposed method outperforms five out of six benchmarked approaches [32], [35], [37], [38], [39], when the JAFFE database is used. When CK database is used it outperforms all the benchmarked approaches.

Table 3. Comparison with State-of-the Art Performance

\begin{tabular}{|c|c|c|c|}
\hline & Feature & JAFFE & CK \\
\hline $\begin{array}{c}\text { Our } \\
\text { approach }\end{array}$ & Patch based Gabor & $\mathbf{8 7 . 2 2 \%}$ & $\mathbf{9 6 . 8 0 \%}$ \\
\hline$[32], 2005$ & Fuzzy integral & $83.2 \%$ & - \\
\hline$[33], 2005$ & Gabor +FSLP & $91.1 \%$ & - \\
\hline$[34] 2006$ & Gabor & - & $93.3 \%$ \\
\hline$[35] 2006$ & KCCA & $77.05 \%$ & - \\
\hline$[36] 2008$ & Gabor+Haar & - & $93.1 \%$ \\
\hline$[37] 2008$ & DCT & $79.3 \%$ & - \\
\hline$[38] 2009$ & Boosted LBP & $81.0 \%$ & $95.1 \%$ \\
\hline$[39] 2010$ & FEETS+PRNN & $83.84 \%$ & $95.87 \%$ \\
\hline
\end{tabular}




\section{CONCLUSION}

This article evaluates the issue of facial expression recognition using facial feature movements. The effectiveness of the method is tested by the recognition performance and comparison with the state-of-the-art performance. The experimental results show significant performance improvements because of the consideration of facial movement features. From the results it is clear that compared to point based Gabor features , Patch based Gabor features shows significant improvement in performance in terms of extraction of regional features, maintaining position information and attaining a better recognition performance. The proposed method can be applied in many applications like patient state detection, intelligent tutoring system and driver fatigue monitoring systems. In future work, the proposed approach can be extended to a video-based FER system by integrating motion information in multiframes with patch-based Gabor features. Recent developments in fields of face recognition, action recognition and the success of using LBP for combining appearance and motion for face analysis from videos has laid the foundation for the use of both appearance and motion features.

\section{ACKNOWLEDGMENTS}

The authors would like to thank the providers of JAFFE Database and Cohn-Kanade AU-Coded Facial Expression Database.

\section{REFERENCES}

[1] B. Fasel and J. Luettin, "Automatic Facial Expression Analysis: A Survey," Pattern Recog., vol. 36, no. 1, (2003), pp. 259-275.

[2] M. Pantic and L. J. M. Rothkrantz, "Automatic Analysis of Facial Expressions: The State of the Art", IEEE Trans. Pattern Anal.Mach. Intell., vol. 22, no. 12, (2000), pp. 1424-1445.

[3] Pantic.M. and Ioannis Patras. -Dynamics of Facial Expression: Recognition of Facial Actions and Their Temporal Segments from Face Profile Image Sequencesll, IEEE transactions on Systems, Man, and Cybernetics-Part B: cybernetics, vol. 36, no. 2, 2006.

[4] V. Bruce, "What the Human Face Tells the Human Mind: Some Challenges for the Robot-Human Interface", Proc. IEEE Int. Workshop Robot and Human Communication, pp. 44-51, 1992

[5] G. Donato, M.S. Bartlett, J.C. Hager, P. Ekman, T.J. Sejnowski, "Classifying Facial Actions", IEEE Trans. Pattern Analysis and Machine Intelligence, Vol. 21, No. 10, pp. 974-989, 1999

[6] I.A. Essa, A.P. Pentland, "Coding, Analysis, Interpretation, and Recognition of Facial Expressions",

IEEE Trans. Pattern Analysis and Machine Intelligence, Vol. 19, No. 7, pp. 757-763, 1997

[7] T. Gritti, C. Shan, V. Jeanne and R. Braspenning, "Local Features based Facial Expression Recognition with Face Registration Errors" 978-1-4244-1/08/2008. IEEE

[8] G. R. S. Murthy and R. S. Jadon, "Effectiveness of Eigenspaces for Facial Expressions Recognition", International Journal of Computer Theory and Engineering, vol. 1, no. 5, (2009) December.
[9] B. Fasel and J. Luttin, "Automatic Facial Expression Analysis: a survey", Pattern Recognition, vol. 36, no. 1, (2003), pp. 259-275.

[10] Z. Zhang, "Comparition between Geometry-Based and Gobor-wavelet-based Facial Expression Recognition Using Multi-layer Perception", Proc. IEEE Int. Conf. Auto. Face Gesture Recog., (1998) April, pp. 454-459

[11] Bouchra Abboud, Franck Davoine, Mo Dang, "Facial expression recognition and synthesis based on an appearance model" 3 May 2004 Elsevier

[12] Ekman, P, Friesen, "Constants across Cultures in the Face and Emotion", J. Pers. Psycho. WV, 1971, vol. 17, no. 2, pp. 124-129

[13] Ekman, P, Friesen, "Facial expressions of emotion: an old controversy and new findings," Philos. Trans. R. Soc. Lond. B, Biol. Sci., vol. 335, pp. 63-69, 1992.

[14] P. Ekman and W.V. Friesen, "Manual for the Facial Action Coding System," Consulting Psychologists Press, 1977.

[15] L. Sirovich and M. Kirby, "Low Dimensional Procedure for Characterization of Human Faces," J. Optical Soc. Am., vol. 4, pp. 519-524, 1987.

[16] K. Mase and A. Pentland, "Recognition of facial expression from optical flow," IEICE Trans. E, vol. 74, pp. 408-410, 1991

[17] I. Essa and A. Pentland, "Facial expression recognition using a dynamic model and motion energy," presented at the Int. Conf. on Computer Vision, 1995

[18] I. Essa and A. Pentland, "Coding, analysis, interpretation, and recognition of facial expressions," IEEE Trans. Pattern Anal. Mach. Intell., vol. 19, no. 7, pp. 757-763, July 1997

[19] P. N. Belhumeur, J. P. Hespanha, and D. J. Kriegman, "Eigenfaces vs. Fisherfaces: recognition using class specific linear projection," IEEE Transactions on Pattern Analysis and Machine Intelligence, vol. 19, no. 7, pp. 711-720, Jul. 1997.

[20] B.A. Draper, K. Baek, M.S. Bartlett, J.R. Beveridge, "Recognizing Faces with PCA and ICA," Computer Vision and Image Understanding: special issue on face recognition, in press.

[21] Y. Tian, T. Kanade, and J. Cohn, "Recognizing action units for facial expression analysis," IEEE Trans. Pattern Anal. Mach. Intell., vol. 23, no. 2, pp. 97-115, Feb. 2001.

[22] Jyh-Yeong Chang and Jia-Lin Chen, "Automated Facial Expression Recognition System Using Neural Networks" Journal of the Chinese Institute of Engineers, Vol. 24, No. 3, pp. 345-356 (2001).

[23] Hong-Bo Deng, Lian-Wen Jin, Li-Xin Zhen, Jian-Cheng Huang, "A New Facial Expression Recognition Method Based on Local Gabor Filter Bank and PCA plus LDA", International Journal of Information Technology Vol. 11 No. 112005

[24] Ceifeng Shan, et al., "Facial expression recognition based on Local Binary Patterns: A comprehensive Study", Elsevier Science Ltd Trans., 2009. 
[25] S. Luiz OLiveira, et al., "2D Principal Component Analysis for face and facial expression recognition", IEEE Trans., 2011.

[26] D. Gabor, "Theory of Communication," J. Institution of Electrical Engineers-Part III: Radio and Comm. Eng., vol. 93, pp. 429-441, 1946.

[27] C.C. Chang and C.J. Lin, "LIBSVM: A Library for Support Vector Machines, 2001," http://www.csie.ntu.edu.tw/cjlin/libsvm,2001.

[28] A. Farahat, A. Ghodsi, and M. Kamel, "An efficient greedy method for unsupervised feature selection," in

Data Mining (ICDM), 2011 IEEE 11th International Conference on, dec. 2011, pp. $161-170$.

[29] C. Cortes and V. Vapnik, "Support-Vector Networks," Machine Learning, vol. 20, pp. 273-297, 1995.

[30] http://www.kasrl.org/jaffe.html.

[31] T. Kanade, J.F. Cohn, and T. Yingli, "Comprehensive Database for Facial Expression Analysis," Proc. IEEE Fourth Int'l Conf. Automatic Face and Gesture Recognition, pp. 46-53, 2000.

[32] W. Yuwen, L. Hong, and Z. Hongbin, "Modeling Facial Expression Space for Recognition,” Proc. IEEE/RSJ Int'l Conf. Intelligent Robots and Systems, pp. 1968-1973, 2005.

[33] G. Guo and C.R. Dyer, "Learning from Examples in the Small Sample Case: Face Expression Recognition,"
IEEE Trans. Systems, Man, and Cybernetics, Part B: Cybernetics, vol. 35, no. 3, pp. 477-488, June 2005.

[34] G. Littlewort, M.S. Bartlett, I. Fasel, J. Susskind, and J. Movellan, "Dynamics of Facial Expression Extracted Automatically from Video," Image and Vision Computing, vol. 24, pp. 615-625, 2006

[35] Z. Wenming, Z. Xiaoyan, Z. Cairong, and Z. Li, "Facial Expression Recognition Using Kernel Canonical Correlation Analysis (KCCA)," IEEE Trans. Neural Networks, vol. 17, no. 1, pp. 233- 238, Jan. 2006.

[36] H.Y. Chen, C.L. Huang, and C.M. Fu, "Hybrid-Boost Learning for Multi-Pose Face Detection and Facial Expression Recognition," Pattern Recognition, vol. 41, pp. 1173-1185, 2008

[37] J. Bin, Y. Guo-Sheng, and Z. Huan-Long, "Comparative Study of Dimension Reduction and Recognition Algorithms of DCT and 2DPCA," Proc. Int'l Conf. Machine Learning and Cybernetics, pp. 407- 410, 2008.

[38] C. Shan, S. Gong, and P.W. McOwan, "Facial Expression Recognition Based on Local Binary Patterns: A Comprehensive Study," Image and Vision Computing, vol. 27, pp. 803-816, 2009.

[39] J.-J. Wong and S.-Y. Cho, "A Face Emotion Tree Structure Representation with Probabilistic Recursive Neural Network Modeling," Neural Computing and Applications, vol. 19, pp. 33-54, 2010. 